\title{
Knowledge and use of contraceptive methods amongst deaf people in Ghana
}

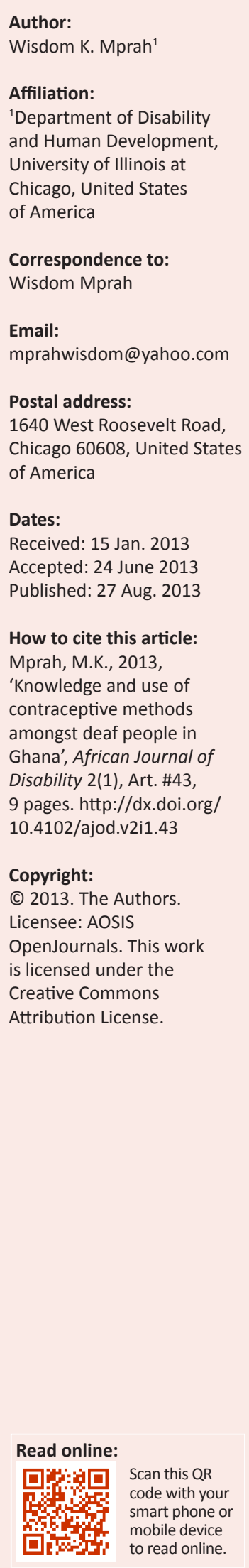

Background: Persons with disabilities in general face serious barriers to sexual and reproductive health (SRH) information and services due to institutional and attitudinal barriers. However, because deaf people have unique communication and linguistic needs, which are often misunderstood or ignored, they face greater barriers than other persons with disabilities. Whilst available data indicated that there is a wide gap between knowledge and usage of contraceptive amongst Ghanaians, little is known about the level of contraceptive knowledge and usage amongst deaf people.

Objectives: The objective of the study was to investigate the level of knowledge and use of contraceptive methods amongst deaf people in Ghana with the aim of understanding their contraceptive behaviour and to improve access.

Method: The study was a participatory SRH needs assessment utilising a two-phase, sequential, mixed methods design. The study included 179 participants, consisting of focus groups with seven executives of Ghana National Association of the Deaf (GNAD), 10 male deaf adults, and 9 deaf female adults. A total of 152 deaf people, made up of students, women, and men participated in a survey, whilst one hearing person served as a key informant.

Results: The findings of the study indicated that of the 13 methods shown in the survey, only three were known to about $70 \%$ of the adults and $60 \%$ of the students. Level of knowledge of the remaining nine methods was low.

Conclusion: Clear and effective policies are needed to guide the provision of SRH information and services for deaf people in Ghana.

\section{Introduction}

Contraceptive knowledge and use are important indicators of access to sexual and reproductive health (SRH) information and services (Ghana Statistical Service, Ghana Health Service \& ICF Macro 2009). Knowledge and usage of contraceptives are also important for determining attitudes towards and awareness about risks associated with pregnancies and sexually transmitted diseases (STDs). Many studies have established that knowledge of methods and sources is a key factor governing effective use of contraceptives (Biney 2011; Longwe, Huisman \& Smits 2012; Small et al. 2009). Having good knowledge reduces misconceptions and fears about contraceptives and creates positive attitudes towards use; generally, the more knowledge people have, the more likely they would accept and use contraceptives (Biney 2011; Longwe et al. 2012; Narzary 2009; Small et al. 2009). For example, Lindstrom and Hernandez (2006) observed that lack of knowledge was commonly cited for unmet needs and limited choice of contraceptives amongst rural or urban migrants in Guatemala. Similarly, Biney (2011) reported that a major reason for not using contraceptives amongst women in Ghana was lack of knowledge or misunderstanding of contraceptives.

It has also been established that engaging in risky sexual activities often led to unintended pregnancies, unsafe abortions, and sexually transmitted infections (STIs) (Biney 2011; Poku 2008). The use of effective contraceptive methods is thus crucial for preventing unintended pregnancies and unsafe abortions. When used properly and consistently, the barrier methods (e.g. condoms) would serve the dual purposes of preventing unintended pregnancies and STIs including HIV, whilst the non-barrier methods would prevent unintended pregnancies (Poku 2008; Small et al. 2009).

Although little is known about the level of knowledge and use of contraceptives amongst persons with disabilities, including deaf people, they are less likely to have knowledge of and use contraceptive methods than persons without disabilities. This is due to information barriers they encounter when accessing services on SRH issues. These barriers are related to ignorance 
about the unique needs of persons with disabilities, negative attitudes, and lack of services tailored to accommodate their needs (Groce 2004; Wilson \& Monaghan 2006; World Health Organization [WHO] 2009). Sexual and reproductive health information is often not provided in accessible formats or tailored to cater for the needs of people with disabilities. For example, the lack of information in accessible forms such as Braille, large print, simple language, pictures, and sign languages, deprive access to those with visual, hearing, and intellectual disabilities (WHO 2009). Low literacy levels amongst persons with disabilities compound the issue of access. Many people with disabilities in developing nations have limited formal education, lack access to SRH education in schools or/and inadequate health literacy after school (Groce 2004; WHO 2009). In addition, their disabilities may limit their chances to interact with their peers, which would be an important opportunity for informal learning about reproduction and sexuality (Haseltine, Cole \& Gray 1993). Moreover, they are rarely included in SRH prevention and outreach programmes due to misconceptions about their sexuality (Job 2004; Wilson \& Monaghan 2006; WHO 2009).

Adolescents with disabilities face particularly severe challenges because it is often difficult for parents, educators, and counsellors to broach the subject or they are perceived as sexually inactive (Job 2004; Prilleltensky 2004). Consequently, many young people with disabilities are not familiar with basic vocabularies about their bodies, cannot describe what is happening to them and are therefore at high risk of SRH problems and sexual exploitation (Groce 2004; WHO 2009).

The available data in Ghana indicate a wide gap between knowledge and practice on issues relating to SRH amongst Ghanaians (Ghana Statistical Service, Ghana Health Service \& ICF Macro 2009). National and local surveys do not have data on persons with disabilities, including people who are deaf and hard of hearing. It is therefore impossible to determine the SRH status amongst people who are deaf and hard of hearing in Ghana. Their situation is likely to be particularly bad as they have limited access to mainstream information (Ministry of Employment and Social Welfare 2000). Not only is it difficult to access such information, it is very likely that informal sources such as friends and family members are inadequate and unreliable. Due to the nature of Ghanaian traditional values, issues of sex are shunned within the family, so families are seldom major sources of sexual and reproductive information (Mensch et al. 1999).

The government and various stakeholders have recently stepped up efforts to increase knowledge on SRH issues. Some key government strategies to address SRH issues in Ghana are the formulation of policies such as the Ghana Population Policy, the Adolescents Reproductive Health Policy and the National HIV / AIDS and STI Policy. Research has also been done to identity groups at high risk for SRH problems, poverty reduction and increased access to information (Hessburg et al. 2007). The main aim of these strategies is to design effective policies, to better understand the SRH behaviour of Ghanaians and to empower individuals to make independent and informed decisions on their sexuality and reproduction.
However, these efforts are unlikely to reach the deaf population in Ghana as the general target is the hearing population. In addition, negative perceptions about deaf people and lack of societal understanding of their concerns have contributed to the disregard of deaf people's needs in SRH policies and service delivery. The few SRH studies and programmes for deaf people have focused mainly on HIV and AIDS with little information on their level of knowledge or use of preventive measures such as contraception. Understanding the level of knowledge and use of contraception is important for policymaking and programme designing for the deaf community. The objective of this study was therefore to investigate the level of knowledge and use of contraceptive methods amongst deaf people in Ghana. The aim was to gain insights into the contraceptive behaviour of the deaf community so as to inform and redirect future action plans and policies. The study proposed the following question: what is the level of knowledge and use of contraceptive methods amongst deaf people in Ghana? In answering this question, the study employed two data collection techniques: focus groups to allow an in-depth exploration of deaf people's views on the subject and to develop a survey instrument, and then implementation of the survey for further exploration of views in the larger deaf community.

\section{Overview of deafness in Ghana}

Very little is known about the demographic characteristics of deaf people in Ghana because population censuses and studies that collect demographic information do not separately identify deaf respondents. However, Ghana National Association of the Deaf reported having 6000 registered members in 2007, of whom 2400 were females and 3600 were males. This number comprises only those who have voluntarily registered with GNAD and presumably represents only a very small portion of the total deaf population in Ghana (Johnson Mahama, pers. comm., 20 March 2010).

Generally, deaf people are distinguished from the hearing population not only by their hearing loss but by their distinct cultural and linguistic characteristics, which include a communication system that is different from spoken language, as well as cultural values that are different from those found amongst hearing people (Sparrow 2005; Tucker 1998). For example, whereas individualism is a dominant cultural pattern in the hearing population in the USA, collectivism is dominant amongst deaf people. Members of the deaf community often consider themselves as a close-knit and interconnected group (Ladd 2003).

In some western countries such as the USA, there are two main deaf cultural perspectives: the medical and the cultural. Whilst the medical refers to deafness as impairment, the cultural model defines deafness in terms of a cultural identity. A traditional impairment perspective would describe deaf persons who do not identify with a separate deaf cultural identity and have been acculturated and assimilated into hearing society (Tucker 1998). They are deaf individuals who are considered clinically deaf and persons with disabilities (Burch 2004; Edwards 2005). Deafness in this case is perceived 
primarily in terms of the underlying medical pathology (Corker 1998; Tucker 1998). On the other hand, deaf people, who subscribe to the cultural identity construction do not consider themselves as persons with disabilities and perceive deafness as a cultural phenomenon rather than a disability. In other words, they define deafness as a linguistic and cultural experience instead of impairment (Padden \& Humphries 2005; Tucker 1998). The inability to hear is essentially parallel to a hearing person's ignorance of the sign language of the deaf community: a social disadvantage rather than a physical disability (Crouch 1997; Tucker 1998).

There is very little documentation about deaf identity in Ghana. My experience with the deaf community and the little that has been written on deaf people in Ghana suggests that the concept of deaf identity is relatively new and limited to the few who have attained some formal education. In fact, the American notion of impairment versus cultural identity construction does not exist in Ghana. Generally, deaf people in Ghana do not differentiate between those who view deafness as a clinical impairment and those who see it as a cultural group. All individuals who have some hearing difficulties are considered members of the deaf community irrespective of one's ability to use the general sign language (GSL), age of onset of deafness, school attended, or family background. However, there are important categories of deaf people within Ghana's deaf community: the hard of hearing, those without formal education who are unable to use the GSL, and those who live in a village called Adamrobe, a community with an unusually large number of deaf people caused by heredity deafness. Deaf people in this community have developed their own sign language, the Adamrobe sign language (ADSL), which is different from the GSL. However, no effort has been made to date to differentiate these groups. Whilst deaf people's membership of ethnic minority has influenced their health status (Jones, Renger \& Firestone 2005) and may also influence their knowledge and use of contraceptives, the study did not investigate if and how this affected deaf people's attitudes towards conceptive use.

\section{Methods}

\section{Study design}

The study was a participatory SRH needs assessment targeting only deaf people who were fluent in the GSL in Ghana. The study utilised a two-phase, sequential, mixed methods design, consisting of three focus groups to assist in the development of a survey and then the implementation of the survey for needs assessment data collection. Review of documents, discussions with a SRH worker, and observations helped to clarify data gathered from the focus groups and survey. The focus groups allowed an in-depth exploration of themes to identify SRH issues that were important for the development of the quantitative (survey) instrument. The survey phase was conducted to document needs related to these themes within the deaf community.

The mixed methods approach facilitates triangulation of data collected on the same issue, which often helps researchers develop a deeper understanding of the issue being investigated (Creswell \& Plano Clark 2007). Triangulation allows the researcher to complement the differing strengths of quantitative and qualitative methods (Creswell \& Plano Clark 2007). For example, Demarest, Holey and Leatherman (1984) used surveys, interviews, and records to assess the educational needs of hospital nursing staff. Participants were randomly assigned to either survey or interviews. A key finding from the study was that the three data collection techniques resulted in different needs. According to the researchers, even though it was more expensive to collect data from multiple sources, they gained a fuller understanding and were better able to interpret the results than if they had relied on only one source. The complexity of SRH issues in Ghana presents similarly complex data collection and interpretation challenges.

\section{Population and sample}

A total of 179 participants were recruited for the study, of which 26 were focus group participants, 152 survey respondents, and one person who served as a key informant. These were the people willing to participate in the study. Also, it was difficult getting eligible volunteers (deaf people with formal education) for the study. All participants except the key informant were members of the deaf community and were considered well informed about issues in the community. Participants comprised all persons who were deaf or hard of hearing and who were fluent in the GSL. Lack of formal education was an exclusion criterion since formal education is required to use the GSL. Communicating with this non-GSL group would have required learning the local language such persons developed to communicate within their communities; this would have been a serious logistical challenge since Ghana is a multilingual society. Moreover, users of GSL were more likely to have used or had attempted to utilise SRH information from education programmes that disseminated material through magazines, posters, online materials, and brochures, and thus were more likely to have better understanding and experiences to explain challenges deaf people encounter when accessing SRH information and services.

Participants were recruited from two communities in Ghana: Tamale, a city from the Northern Zone and Accra from the Southern Zone of Ghana. The intent in selecting these communities was to sample respondents with diverse characteristics so that views from people with different perspectives on the topic could be represented. Tamale and Accra represent the northern and southern sectors of the country, which reflect important differences in culture and socio-economic development. The northern sector is generally poor and characterised by poorly developed infrastructure and harsh climatic conditions as compared with the southern sector (Berry 1995; National Population Council 1994).

Specific locations where participants were recruited from in the two cities were a deaf senior high school, deaf churches, and a deaf centre. Whilst focus group participants were recruited from two deaf churches and a deaf centre in Accra, 
recruitment of survey respondents was conducted in a deaf high school and three deaf churches. These locations were selected in order to increase the likelihood of identifying deaf people who had formal education and knowledge of the GSL. The key informant was recruited from one of the SRH centres. The informant had done a study on HIV and AIDS with the deaf community and so he was familiar with the deaf community. Recruitment was done through announcements that included information sessions about the study and eligibility requirements. Informed consent was obtained from all participants before recruitment.

Of the 152 respondents who completed the survey, nearly twothirds were from the deaf senior high school. Respondents from Accra and Tamale represented $28 \%-11.2 \%$ of the survey sample, respectively. More survey participants were chosen from the senior high school than from Accra and Tamale because it is the only public deaf senior high school in Ghana. The senior high school admits students from all over the country and has a diverse deaf population in terms of economic and socio-cultural characteristics. As such, their views were likely to be representative of the adolescent deaf population in Ghana. The inclusion of adolescents was important since this age group has been found to have more SRH problems than other segments of the population (National Population Council 1994). Moreover, it was difficult recruiting deaf people with formal education from Tamale because many of the educated had migrated to the southern part of Ghana in search of jobs and better educational opportunities. This is a longstanding problem for Ghanaians generally; the poor conditions in the north have triggered a general migration of people from the north to the south (Berry 1995).

Efforts were made to ensure that females were equally represented since young girls have been found to be more at risk for SRH problems than boys (National Population Council 1994). Of the students recruited from the senior high school, 44 were females, although female students constituted only 93 of the 343 student population. In the study, respondents from Accra and Tamale (aged 22 years and above) are referred to as the 'adult population' and those from the deaf senior high school (aged 18-22 years) as 'students' or 'adolescents' in the balance of reporting.

\section{Sampling strategy}

The sampling procedure used for selecting participants for this study was purposive, targeting only persons considered knowledgeable of issues affecting the deaf community.

Focus group participants were recruited from a deaf high school, three deaf churches, and a deaf centre. Prospective focus group participants were contacted through text messages and emails. Written scripts of the recruitment announcements were developed in English but were communicated to participants via the GSL at introductory meetings. After contacting prospective participants, arrangements were made to meet the males and the females at two different locations to discuss the focus group procedures, their remuneration, and issues concerning their privacy and confidentiality. Ten out of the 12 contacted agreed to participate. Of the 15 females contacted, 9 agreed to participate. Seven of 10 GNAD executives agreed to participate. After obtaining permission from the SRH unit head, the key informant was recruited.

Recruitment of survey respondents was conducted through announcements that included information about the study, eligibility requirements, and an invitation to volunteers to go through screening and the informed consent process at present dates and times. On the screening day, those who qualified to participate were asked to sign the informed consent forms. In the deaf high school, verbal permission was sought from the head of the school before recruitment began, and a notice was sent to teachers and students about the study. The students were met during their lunch where additional details about the study and eligibility requirements were provided.

\section{Reliability and validity of the data}

As indicated previously, the sampling procedure used for selecting participants was purposive. One of the decisions guiding the use of purposive sampling was to sample participants who had adequate knowledge on SRH issues affecting the deaf community and whose views could best represent the views of the community. Using these participants maximised the utility of the data by minimising input from respondents lacking any perspectives on the concerns of the community.

Data from the focus groups, key informant interviews, personal observations, and survey were compared and combined. Triangulating data from multiple sources thus increased the validity of conclusions and often provided a more sophisticated interpretation of the data. For example, the focus groups facilitated in-depth exploration of the contextual nuances that clarified the survey data. As Creswell and Plano Clark (2007) observed, triangulation in mixed methods, which involves obtaining different but complementary data on the same issue, makes it possible for researchers to get a better understanding of the issue being investigated. The main aim of triangulation is to bring together the differing strengths of quantitative and qualitative methods in order to overcome the weaknesses of each of these methods (Creswell \& Plano Clark 2007).

\section{Data collection and analysis}

\section{Focus groups}

Three focus groups were conducted: (1) the executives group consisting of seven executive members of GNAD, all of whom were males, (2) the adult male group with 10 members, and (3) the adult female group with nine members. Members were selected based on their knowledge of issues that affect the deaf community - they were considered opinion leaders in the deaf community. Those selected included current executives of GNAD, past executives of GNAD, and people serving on committees in the deaf churches. 
The focus group guide consisted of open-ended questions and elicited information on participants' views concerning access to SRH services and information. Issues discussed were: (1) sources of information, (2) knowledge of SRH problems in the deaf community, (3) SRH experiences and needs of deaf people, (4) ways to correct problems deaf people encounter when accessing information and services on SRH issues, (5) key related issues in the deaf community, and (6) the role of GNAD in the provision of information and services on SRH issues. Video and audio recordings were used to record proceedings from the focus groups with participants' permission. Whilst the men's and the executives' focus groups were conducted by a male research assistant, the females' focus group was conducted by a female research assistant. The researcher assisted the research assistants when probes were needed for clarification or when the discussions went off-topic. The research assistants and researcher were all native signers and so all the focus groups were conducted in the GSL.

The transcribed data from the three focus groups were analysed separately in order to differentiate the responses of the three categories of participants: leaders of the deaf community, male participants, and female participants. Focus group videotapes were converted to DVDs using Adobe premiere video software. Both the DVDs and the voice recordings were transcribed to text format. The transcription of the data from the DVDs was done in two steps: 'partial' transcription and full transcription.

The first step ('partial' transcription) involved viewing the DVDs from all the focus groups to identify and transcribe into word documents concerns that were raised by participants. This was an abridged version of the discussions, consisting of only the group discussion material needed for the development of the survey. Since a verbatim transcription of the DVDs would require significant time and delay the development of the survey, an abbreviated procedure was employed. The second step was a 'full' transcription of the videotapes. The full transcription represented the data from the focus groups that were used to complement survey results from the final survey sample.

\section{Survey}

Transcripts from the focus groups video and audio, two existing surveys - the 2003 Ghana Demographic and Health Survey (GDHS) and a survey on SRH status amongst persons with disabilities in Ghana - and two reports on adolescent reproductive health in Ghana were used to develop the survey.

The issues included in the survey were problematic areas drawn from the literature and additional concerns identified in the analysis of the focus group transcripts: experiences of deaf persons with SRH providers, the role of GNAD in the provision of SRH information and services, and suggestions on how to improve access to information and services on $\mathrm{SRH}$ issues.
The final survey explored issues relating to factors that influence visits to SRH centres, organisations providing SRH services, SRH problems amongst deaf people, sources of information on SRH issues, level of knowledge on STDs and pregnancy, contraception knowledge and use, and importance and satisfaction ratings of SRH issues and services.

Based on advice from the GNAD, all the survey interviews were conducted in groups with the exception of the Tamale participants who were interviewed individually. Each interview session involved gathering participants in a single room, distributing surveys, and providing instructions. Research staff provided assistance and answered questions. Some of the items were written on blackboards and flip charts. Writing items on the blackboards and flip charts made it easier to explain items to all the respondents at the same time without having to go round to assist each respondent who needed help. The survey was conducted by the researcher and his two research assistants in the GSL.

Basic descriptive statistics were used to analyse and summarise the survey data. Responses to the survey items were entered into an Statistical Product and Service Solutions (SPSS) data file, and cross tabulations and chi-square statistics were computed to compare response differences across age and gender groups.

\section{Ethical issues}

The study was approved by the University of Illinois at Chicago's (UIC) Institutional Review Board (IRB). As stated previously, verbal permission was sought from the head of the deaf high school before recruitment. Informed consent was obtained from all participants using the GSL. In the case of the focus groups, the men were met twice prior to initiating the focus group sessions. During the first meeting, it was agreed that the informed consent process and the focus group should not be held on the same day in order to allow more time for the informed consent process. Forms were distributed to the participants to study at home; 10 of the 12 who attended the first meeting returned the forms to the second meeting. The meeting with the female focus group was scheduled separately in another church. As with the men's group, the informed consent process was completed prior to beginning the focus groups. Informed consent and the focus group were scheduled on the same day for the executives since they were dispersed across different cities and holding multiple meetings was logistically difficult.

Informed consent for survey participants was conducted in groups. Forms were distributed to prospective participants at meetings in the deaf churches, deaf centres, and a classroom (in the case of the students) when the study was being announced. Prospective volunteers interested in participating returned the forms on the date scheduled for the consent process and recruitment. The key informant was also given the consent form for review in advance of a second meeting during which he was taken through the consent process and the consent subsequently given. 
The informed consent process included giving information about the expected duration of the survey, how much they would be paid, and confirming that participation was completely voluntary and their decision not to participate would not adversely affect their relationship with the investigator. In addition, they were assured that they were not obliged to answer all questions, had the right to withdraw from the study at any time, and that their names would not be identified in the data.

Focus group volunteers were told that the groups involved video and audio taping; whilst the male group agreed to be videotaped, they thought they would feel more comfortable if the deaf community were responsible for the recordings. Thus the researcher did the videotaping and the group chose a pastor working with the deaf church to narrate and audio record what they signed. Similarly, the women agreed to have a deaf person do the videotaping. The executives group was videotaped as well and one of the participants did the audiotape recording.

\section{Results}

A unique feature of the study was the use of the results from qualitative methods to develop the quantitative (survey) instrument. Issues that were raised during the focus groups and had some bearing on the provision of accessible SRH information and services for the deaf community were used to develop the survey. Additionally, issues that generated disagreements or differing perspectives and required the gathering of additional information were included in the survey.

\section{Focus groups results}

Participants discussed the level of knowledge and use of contraceptive methods amongst deaf people in Ghana. Their responses suggest that contraceptive knowledge and use amongst deaf people were low. Some of the participants claimed that deaf people lack knowledge about risks and therefore engaged in risky sexual behaviours, suggesting that deaf people engaged in unprotected sexual activities, that is, do not use contraceptive methods. For example, some participants from the executives' group were of the view that deaf people were largely unaware about the consequences of unsafe sex, which according to them, has resulted in many $\mathrm{SRH}$ problems amongst deaf people. Teenage pregnancy, gonorrhea, syphilis, miscarriage, abortion, and HIV or AIDS were some of common SRH problems identified by the participants.

This perspective on deaf people's lack of knowledge was largely consistent with views from the women's and men's groups. A female participant (31 years), for instance, claimed that 'deaf people engaged in risky sexual behaviors which often resulted in unintended pregnancies', a claim which was supported by another female participant (43 years) who claimed the problem was particularly high amongst adolescents and those who had no formal education.
This pessimistic position reflected in the comments of the women was corroborated by some of the male participants. A male participant (45 years) indicated that deaf people were more likely to engage in risky SRH behaviours because of lack of information on how to protect themselves. Another male participant (29 years), described how deaf people's ignorance about the consequences of unsafe sex has resulted in deaf people engaging in practices that led to many SRH problems:

'There are many diseases and reproductive problems in the deaf community because deaf people are ignorant about safe sex. For example, deaf people suffer from HIV/AIDS, gonorrhea, teenage pregnancies, abortion, and unwanted pregnancies because they engage in risky sexual behaviour. Deaf people do not also have knowledge on the use of condoms as a safe measure against STIs and STDs. So some deaf behave in a way that can lead to health problems, for example, some still share blades and engage in unprotected sex.'

However, this negative perception about deaf people's level of knowledge was not widely accepted by two male focus group participants. For example, one participant (56 years) commented that 'many deaf people know how to take care of themselves', whilst another (48 years) stated that 'some deaf people have knowledge on the use of condoms as a safe measure against STIs and STDs'. The level of disagreement suggested the importance of collecting additional data via surveys on the level of knowledge and use of contraception amongst deaf people.

\section{Survey results}

A summary of the level of knowledge of contraceptive methods amongst the survey respondents was drawn up (Table 1). As indicated in the table, knowledge of modern contraceptive methods was not widespread amongst respondents; for example, respondents tended not to be familiar with modern methods of contraception other than condoms, pills, and injections. However, knowledge of traditional methods was high.

TABLE 1: Percentage indicating knowledge of contraceptive methods by age and gender.

\begin{tabular}{|c|c|c|c|c|c|c|c|c|}
\hline \multirow[t]{2}{*}{ Method } & \multicolumn{2}{|c|}{ Students (M) } & \multicolumn{2}{|c|}{ Adults (M) } & \multicolumn{2}{|c|}{ Students (F) } & \multicolumn{2}{|c|}{ Adults (F) } \\
\hline & $n$ & $\%$ & $n$ & $\%$ & $n$ & $\%$ & $n$ & $\%$ \\
\hline \multicolumn{9}{|l|}{ Modern methods } \\
\hline Pills & 31 & 64.6 & 21 & 53.8 & 24 & 54.5 & 10 & 47.6 \\
\hline Injections & 36 & 70.8 & 29 & 74.4 & 24 & 54.5 & 15 & 71.4 \\
\hline Condoms & 31 & 75.0 & 30 & 76.9 & 23 & 52.3 & 13 & 61.9 \\
\hline $\begin{array}{l}\text { Emergency } \\
\text { contraceptives }\end{array}$ & 5 & 10.4 & 15 & 38.5 & 25 & 56.8 & 10 & 47.6 \\
\hline IUDs & 11 & 22.9 & 6 & 15.4 & 10 & 22.7 & 11 & 52.3 \\
\hline Implants & 14 & 29.2 & 7 & 17.9 & 17 & 38.6 & 5 & 23.8 \\
\hline Jelly/foam & 12 & 25.0 & 11 & 28.2 & 10 & 22.7 & 6 & 28.6 \\
\hline Female sterilisation & 16 & 33.3 & 4 & 10.3 & 10 & 22.7 & 5 & 23.8 \\
\hline Male sterilization & 17 & 35.4 & 8 & 20.5 & 7 & 15.9 & 6 & 28.6 \\
\hline \multicolumn{9}{|l|}{ Traditional methods } \\
\hline Withdrawal & 25 & 52.1 & 22 & 56.4 & 19 & 43.2 & 11 & 52.4 \\
\hline $\begin{array}{l}\text { Periodic abstinence/ } \\
\text { rhythm }\end{array}$ & 26 & 54.2 & 16 & 41.0 & 23 & 52.3 & 16 & 76.1 \\
\hline
\end{tabular}

$\mathrm{M}$, male; $\mathrm{F}$, female; $n$, number of respondents who responded to the item. 
Adults tended to have higher levels of familiarity than students and there was a tendency for knowledge to be gender specific, with males generally more familiar with male-oriented contraceptive methods and females more familiar with methods relevant to females. However, none of the chi-square tests were significant across age and gender for each method.

The current use of contraceptives as reported by respondents was also analysed (Figure 1). As expected, contraceptive usage was generally low for all respondents. There were no statistically significant gender differences in contraceptive use but there was an age effect, with adults reporting more contraceptive use than students $(46.7 \%$ versus $35.9 \%$, $\left.x^{2}=10.125, d f=2, p=007\right)$. However, the age effect might simply reflect the large proportion of students who chose 'not applicable' (30.1\% students versus $12.5 \%$ adults, not shown in the diagram). The low contraceptive usage amongst respondents seems to be consistent with perspectives by focus group participants that deaf people engaged in unprotected sex.

Regarding the reasons for using any contraceptive methods, fear of contracting STDs, including HIV or AIDs seems to be the major reason amongst both adults and students, as seen in the summary of respondents' reason for using contraceptives across gender and age (Table 2).

There were no age or gender effects for fear of HIV or AIDS or STDs as a reason for contraceptive use. Although the tendency to rate fear of pregnancy was highest amongst adult females, there was no statistically significant gender difference in the ratings. Age was a more important factor

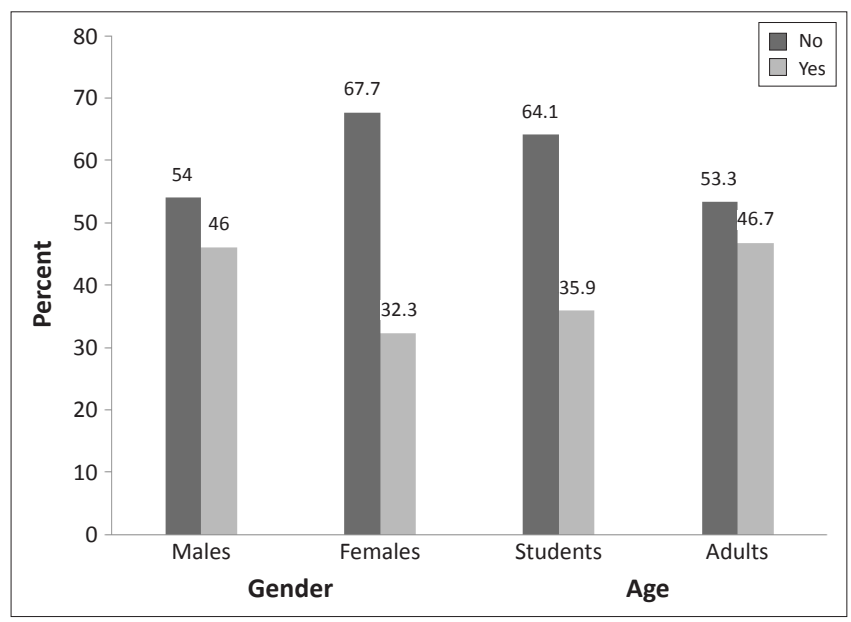

FIGURE 1: Percentage sample of current use of contraceptives by age and gender

TABLE 2: Percentage of sample citing reasons for using contraceptives by age and gender.

\begin{tabular}{|c|c|c|c|c|c|c|c|c|}
\hline \multirow[t]{2}{*}{ Reasons } & \multicolumn{2}{|c|}{ Students (M) } & \multicolumn{2}{|c|}{ Adults (M) } & \multicolumn{2}{|c|}{ Students (F) } & \multicolumn{2}{|c|}{ Adults (F) } \\
\hline & $n$ & $\%$ & $n$ & $\%$ & $n$ & $\%$ & $N$ & $\%$ \\
\hline Fear of HIV or AIDS & 23 & 62.2 & 24 & 75.0 & 22 & 62.9 & 8 & 47.1 \\
\hline Fear of pregnancy ${ }^{a}$ & 5 & 33.3 & 7 & 33.3 & 3 & 21.4 & 5 & 62.5 \\
\hline Fear of other STDs & 2 & 9.1 & 4 & 16.7 & 4 & 28.6 & 1 & 16.7 \\
\hline
\end{tabular}

$\mathrm{M}$, male; F, Female; $n$, number of respondents who responded to the item; STD, sexually $\mathrm{M}$, male; $\mathrm{F}$, Female; $n$, number
transmitted disease; ${ }^{2}$, across age. $p<0.05$. in ratings of fear of pregnancy, with students (11.1\%) less likely to rate it as an important reason compared to adults $(24.5 \%)\left(x^{2}=10.125, d f=2, p=006\right)$. Female students were the least concerned about pregnancy, contradicting findings on a study on adolescent reproductive health in Ghana, which reported that the primary motivation for contraceptive use amongst adolescents was fear of pregnancy, not of STDs (Hessburg et al. 2007).

It should be noted that the survey had no items that elicited information on whether respondents were married or in a sexual relationship at the time of the survey. Contraceptive use is unnecessary if the respondent was not married or not sexually active. Thus the low rate of contraceptives usage amongst respondents, particularly amongst the students, cannot be construed as lack of access to contraceptive methods, having a negative attitude towards contraceptive methods, or fear of embarrassment for reporting contraceptive use.

\section{Discussion}

This study explored deaf people's level of knowledge and use of contraceptive methods and found that whilst deaf people's level of knowledge and use of contraceptives was generally low, contraceptive knowledge appeared to depend on the type of contraception, age and sex. This finding is somewhat consistent with findings from Poku's (2008) general survey, which found that the level of contraceptive knowledge amongst people with disabilities in Ghana was low.

A comparison between findings of the current study and findings of the GDHS, a national survey to provide a broad understanding of the demographic characteristics and health status of the general population in Ghana (Ghana Statistical Service, Ghana Health Service \& ICF Macro 2009), indicated that contraceptive knowledge amongst deaf people was generally lower than that of the general population in Ghana. However, care must be taken when comparing the results of the current study with other groups in order not to harm any of the groups involved. For example, the low level of knowledge of contraceptive methods amongst deaf people in the current study compared to the general population should be interpreted with caution because the two populations are not the same. Deaf people's low level of knowledge should not be stereotyped and attributed to their hearing loss; rather it should be seen as resulting from barriers they encounter. Comparing the two populations in the same study may provide a better picture, but the GDHS and other national studies do not report statistics on the deaf population, so it is impossible to make such comparisons. Pollard (1992) warned of the need to insure that when differences are observed in comparing deaf and hearing people, such differences should not lead to conclusions that will be derogatory or demeaning to either group.

The finding that knowledge on condoms, withdrawal method, and pills was high amongst deaf people is consistent with findings from some previous studies amongst deaf people. For example, Job (2004) found that the most commonly 
reported type of contraception amongst deaf adolescents were withdrawal, condoms, and oral contraception. The finding in the current study is also somewhat consistent with findings in the general population in Ghana. Findings from the GDHS indicated that the level of knowledge of condoms, injectables and pills was higher than other methods. These findings suggest some similarities between the deaf population and hearing population in terms of contraceptive behaviour. However, deaf people have unique characteristics that will present unique challenges in addressing their contraceptive needs. For example, communication barriers present serious difficulties for deaf people when obtaining information from the major sources of information such as health professionals, media, and reading materials. It should be noted that the level of knowledge of these methods is high probably because they seem to be the most popular methods and obtaining them can be done easily from any pharmacy and chemist shop without prescription.

Survey data in the present study indicated that the motivation to use contraception was fear of contracting STDs, including HIV or AIDS, rather than pregnancy. The finding is consistent with the general reproductive behaviour of Ghanaians (Ghana Statistical Service, Ghana Health Service \& ICF Macro 2009). The GDHS reported that the main reasons for not using contraceptive methods amongst married women in Ghana was fertility related: the desired to have more children or infecundity (Ghana Statistical Service, Ghana Health Service \& ICF Macro 2009). This, however, contradicts a study on adolescents' reproductive health in Ghana which indicated that adolescents would use contraceptives for preventing pregnancies rather than for preventing HIV or AIDS (Hessburg et al. 2007). There are no obvious reasons for these differences. It might be that participants in the current study perceived HIV or AIDS as a greater personal danger than pregnancy. Hessburg et al. (2007) study indicated that participants thought they could identify a person with HIV or AIDS by physical symptoms. This perception may have influenced their decision on contraceptive use; they probably thought they could easily identify a person with HIV or AIDS from the person's physical appearance and then make a decision about whether to use any preventive methods. These findings suggest there are differences in perceptions and attitudes between hearing and deaf people, and thus the need for group-specific intervention strategies.

A unique feature of the study was the use of the results from qualitative methods to develop the quantitative (survey) instrument. Integrating two data collection techniques (focus groups and a survey) from two divergent research traditions in a single study presented opportunities for tapping the strengths of the two approaches, and at the same time, compensating for their weaknesses. It also presented some challenges. First, the focus groups facilitated the development of the survey instrument - it assisted in formulating survey questions. Second, combining the two data collection techniques provided a better understanding of the topic under investigation and helped to overcome the complex data collection and interpretation challenges inherent in SRH research. Analysing deaf people's level of knowledge and use of contraceptives from differing methodological perspectives provided better and deeper insights into the subject. Third, the use of the two methods strengthened the validity of the findings; the fact that the findings of the focus groups and survey point to the same conclusion suggest that the findings are, to a large extent, valid.

Notwithstanding these benefits, integrating qualitative and quantitative methods has serious limitations, notably comparability of the results from the two research methods. The focus group data are necessarily interpretative and typically dependent on the context in which the actions take place and thus are not amenable to quantification which is the basis of quantitative (survey) data. This presented a methodological difficulty in reaching a common conclusion without some simplifications.

\section{Policy and programmatic implications}

The findings of the study have important implications for policy making and programme designing. Deaf people have unique communication needs which are often ignored in SRH policy making, programme designing, and service delivery. The neglect of these needs has created barriers and hindered accessibility to SRH information and services to the deaf community. For example, most educational campaigns on SRH issues in Ghana are conducted primarily through the mass media and educational materials such as posters and brochures (Awusabo-Asare et al. 2006; Ghana Statistical Service, Ghana Health Service \& ICF Macro 2009). There are, however, important limitations to media-based dissemination to the deaf community because television broadcasts are not captioned or translated in the GSL. More limiting is the fact that these programmes reach few people since many in the deaf community do not possess television sets. The print media and internet are likewise less accessible to the deaf because of their costs and the limited English reading skills in the population. This lack of accessible information is compounded by the high illiteracy rate in the deaf community which prevents access to commonly used print materials such as newspapers, magazines, leaflets, brochures, posters and billboards.

Thus any successful SRH programme for the deaf community must address their communication concerns. Possible solutions could include having the Ghana National Association of the Deaf (GNAD) appeal to all television stations to adapt their programmes with sign language interpretation or by providing subtitles. Secondly, all SRH education outreach programmes for the deaf community should be undertaken in collaboration with GNAD so that GNAD could provide technical assistance and help with the provision of sign language interpreters.

Finally, whilst the survey found that knowledge of some contraceptive methods such as pills, injectables and condoms was generally high, awareness of many other methods was 
not widespread. In particular, understanding of emergency contraceptives should be improved because it is an important method following unprotected sex (Awusabo-Asare et al. 2006). Also, since the motivation to use contraceptive methods appears to come from fear of contracting HIV or AIDS rather than pregnancy, investing in HIV or AIDS campaigns can have indirect but far reaching effects on the reduction of unintended pregnancies.

\section{Conclusion}

The study findings highlighted key issues relating to deaf people's level of knowledge and use of contraceptive methods, which are relevant for the design of deaf friendly policies and programmes. The study findings suggest that serious challenges lie ahead in the provision of deaf-friendly SRH information and services for the deaf community in Ghana. This demands clear and effective policies to guide the provision of information and services. Moreover, to the extent that cultural considerations are fundamental to SRH planning, the matter of deaf culture and its influence on the behaviour of deaf people must be considered. In other words, further research is needed to understand the relative importance of deaf people's cultural identity and communication barriers for their vulnerability to SRH problems.

\section{Acknowledgements}

I would like to thank the National Association of the Deaf, and the entire deaf community in Ghana and individuals, who in diverse ways, contributed to the success of this project. The financial assistance from the International Fellowships Programme and the Department of Disability Human Development, University of Illinois at Chicago, contributed significantly to the completion of this project. My sincere thanks also go to my doctoral committee.

\section{Competing interests}

The author declares that he has no financial or personal relationship(s) that may have inappropriately influenced him in writing this article.

\section{References}

Awusabo-Asare, K., Biddlecom, A., Kumi-Kyereme, K. \& Patterson, K., 2006, 'Adolescent sexual and reproductive health in Ghana: Results from the 2004 National Survey of Adolescents,' Occasional Report 22, viewed 09 September 2008, from http://www. guttmacher.org/pubs/2006/06/08/or22.pdf

Biney, A.A.E., 2011, 'Exploring contraceptive knowledge and use among women experiencing induced abortion in the Greater Accra Region, Ghana', viewed 24 May 2013, from http://www.ug.edu.gh/rips/pub/Research_Article_by_Adriana_ AE_Biney.pdf

Berry, L.B., 1995, Ghana, a country study, US Government Printing Office, Washington. Burch, S., 2004, Signs of resistance: American deaf cultural history, 1900 to World War II, New York University Press, New York.

Corker, M., 1998, Deaf and disabled or deafness and disabled?, Open University Press, Philadelphia.

Creswell, J.W. \& Plano Clark, V.L., 2007, Designing and conducting mixed methods research, Sage Publications, Thousand Oaks.
Crouch, A.R., 1997, 'Letting the deaf be deaf: Reconstructing the use of cochlear implants in prelingually deaf children', The Hastings Center Report 27(4), 14-21, viewed 20 in prelingually deaf children', The Hastings Center Report 27(4), 14-21, viewed 20
September 2008, from www.cinahl.com/cgi-bin/refsvc?jid=733\&accno=1998009348

Demarest, L., Holey, L. \& Leatherman, S., 1984, 'The use of multiple methods to assess continuing education needs', paper presented at the annual meeting of the Evaluation Network, October 1984, San Francisco.

Edwards, R.A.R., 2005, 'Sound and fury; Or, much ado about nothing? Cochlear implant in the historical perspective', Journal of American History 92(3), 892-920, viewed 10 September 2008, from http://jah.oxfordjournals.org/content/92/3/892.ful

Ghana Statistical Service (GSS), Ghana Health Service (GHS) \& ICF Macro, 2009, 'Ghana demographic and health survey 2008', GSS, GHS, and ICF Macro, Accra, Ghana.

Groce, N.E., 2004, 'Adolescents and youth with disability: Issues and challenges' Asia Pacific Disability Rehabilitation Journal 15(2), 13-32, viewed 11 June, 2010, from http://www.aifo.it/english/resources/online/apdrj/apdrj204/adolescent.pdf

Haseltine, F.P., Cole, S.S. \& Gray, D.B., 1993, Reproductive health issues for persons with physical disability, Paul H. Brookes, Baltimore.

Hessburg, L., Awusabo-Asare, K., Kumi-Kyereme, K., Nerquaye-Tetteh, J.O., Yankey, F., Biddlecom, A. et al., 2007, 'Protecting the next generation in Ghana: New evidence on adolescent sexual and reproductive health needs,' viewed 10 June 2009, from http://www.guttmacher.org/pubs/2007/11/13/PNG_Ghana.pdf

Job, J., 2004, 'Factors involved in the ineffective dissemination of sexuality information to individuals who are deaf or hard of hearing', American Annals of Deaf 149(3) 264-273, viewed 11 May 2007, from http://web.ebscohost.com.proxy.cc.uic.edu/ ehost/pdfviewer/pdfviewer?sid=755f43d5-4bf0-4801-a582-36a9cab8f3fc\%40ses sionmgr10\&vid $=4 \&$ hid $=25$

Jones, G.E., Renger, R. \& Firestone, R., 2005, 'Deaf community analysis for health education Priorities' Public Health Nursing 22(1), 27-35, viewed 20 March 2008 from http://web.ebscohost.com.proxy.cc.uic.edu/ehost/pdfviewer/pdfviewer?sid= e76d4591-3d59-4c73-abb4-684b2b5a11c0\%40sessionmgr11\&vid=4\&hid=25

Ladd, P., 2003, Understanding deaf culture: In search of deafhood, Multilingual Matters, Clevedon.

Lindstrom, D.P. \& Hernández, C.H., 2006, 'Internal migration and contraceptive knowledge and use in Guatemala', International Family Planning Perspectives 32(3): 146-153. http://dx.doi.org/10.1363/3214606, PMid:17015244

Longwe, A., Huisman, J. \& Smits, J., 2012, 'Effects of knowledge, acceptance and use of contraceptives on household wealth in 26 African countries', viewed 31 May 2013, from http://www.ru.nl/nice/workingpapers

Mensch, B.S., Bagah, D., Clark, H.W. \& Binka, F., 1999, 'Changing nature of adolescence behavior in the Kassena-Nankana district of Ghana', Studies in Family Planning 30(2), 95-111. http://dx.doi.org/10.1111/j.1728-4465.1999.00095.x, PMid:16617544

Ministry of Employment and Social Welfare, 2000, Ghana national disability policy document, Delaram, Accra, Ghana.

National Population Council, 1994, 'National population policy', Ghana National Population Council, Accra, Ghana.

Narzary, P.K., 2009, 'Knowledge and use of contraception among currently married, adolescent women in India', Studies on Home Community Science 3(1), 43-49, viewed 29 May 2013, from http://www.krepublishers.com/02-Journals/S-HCS/ HCS-03-0-000-09-Web/HCS-03-1-000-09-Abst-PDF/HCS-03-1-043-09-067 Narzary-P-K/HCS-03-1-043-09-067-Narzary-P-K-Tt.pdf

Padden, C. \& Humphries, T., 2005, Inside deaf culture, Harvard University Press, Cambridge. PMCid:PMC548320

Poku, K.A., 2008, 'Sexual and reproductive health status and HIV/AIDS and STI-related knowledge, attitude, and behaviors among persons with disabilities in Ghana', Unpublished report, Ghana AIDS Commission.

Pollard, R.Q., 1992, 'Cross-cultural research ethics in the conduct of deafness research', Rehabilitation Psychology 37(2), 87-99, viewed 23 May 2010, from http://www. urmc.rochester.edu/ncdhr/documents/cross-cultural-ethics-in-the-deafnessresearch.pdf?redir=128.151.10.65/ncdhr/documents/cross-cultural-ethics-in-thedeafness-research.pdf

Prilleltensky, O., 2004, Motherhood and disability: Children and choices, Palgrave Macmillan, New York. http://dx.doi.org/10.1057/9780230512764

Small, E., Weinman, M., Buzi, R.S. \& Smith, P., 2009, 'Risk factors, parental communication, self and peers' beliefs as predictors of condom use among female adolescents attending family planning clinics', Journal of HIV/AIDS \& Social Services 8(3), 251-268, viewed 10 April 2013, from http://www.tandfonline.com/doi/abs/10.1080/15381 500903130504 ? journalCode=whiv20\#preview

Sparrow, R., 2005, 'Deaf culture: The case of cochlear implants', The Journal of Political Philosophy 13(2)135-152, viewed 12 June 2009, from http://www.neiu. edu/ gmoreno1/Special_Education_Courses_with_Dr._Moreno/Module_Nine_ files/ActivitySix.pdf

Tucker, P.B., 1998, 'Deaf culture, cochlear implants, and elective disability', The Hastings Center Report 28(4), 6-14, viewed 14 March 2009, from http://findarticles.com/p/ articles/mi_go2103/is_n4_v28/ai_n28711241/

Wilson, A. \& Monaghan, L., 2006, 'HIV/AIDS and the deaf community', International Journal of Deaf Studies 22(1), 1-10.

World Health Organization, 2009, 'Promoting sexual and reproductive health for persons with disabilities: WHO/UNFPA guidance note', viewed 19 June 2010, from http://www.unfpa.org/webdav/site/global/shared/documents/publications/2009/ srh_for_disabilities.pdf 